\title{
Novel insights into the dynamics behavior of glucagon-like peptide-1 receptor with its small molecule agonists
}

Girdhar, Khyati; Dehury, Budheswar; Kumar Singh, Mahender; Daniel, Vineeth P.; Choubey, Abhinav; Dogra, Surbhi; Kumar, Sunil; Mondal, Prosenjit

Published in:

Journal of Biomolecular Structure and Dynamics

Link to article, DOI:

$10.1080 / 07391102.2018 .1532818$

Publication date:

2019

Document Version

Peer reviewed version

Link back to DTU Orbit

Citation (APA):

Girdhar, K., Dehury, B., Kumar Singh, M., Daniel, V. P., Choubey, A., Dogra, S., Kumar, S., \& Mondal, P. (2019). Novel insights into the dynamics behavior of glucagon-like peptide-1 receptor with its small molecule agonists. Journal of Biomolecular Structure and Dynamics, 37(15), 3976-3986.

https://doi.org/10.1080/07391102.2018.1532818

\section{General rights}

Copyright and moral rights for the publications made accessible in the public portal are retained by the authors and/or other copyright owners and it is a condition of accessing publications that users recognise and abide by the legal requirements associated with these rights.

- Users may download and print one copy of any publication from the public portal for the purpose of private study or research.

- You may not further distribute the material or use it for any profit-making activity or commercial gain

- You may freely distribute the URL identifying the publication in the public portal 


\title{
Journal of Biomolecular Structure and Dynamics
}

\section{Novel insights into the dynamics behavior of Glucagon-Like Peptide-1 Receptor with its small molecule Agonists}

\author{
Khyati Girdhar, Budheswar Dehury, Mahender Kumar Singh, Vineeth $\mathbf{P}$ \\ Daniel, Abhinav Choubey, Surbhi Dogra, Sunil Kumar \& Prosenjit Mondal
}

To cite this article: Khyati Girdhar, Budheswar Dehury, Mahender Kumar Singh, Vineeth P Daniel, Abhinav Choubey, Surbhi Dogra, Sunil Kumar \& Prosenjit Mondal (2018): Novel insights into the dynamics behavior of Glucagon-Like Peptide-1 Receptor with its small molecule Agonists, Journal of Biomolecular Structure and Dynamics, DOI: 10.1080/07391102.2018.1532818

To link to this article: https://doi.org/10.1080/07391102.2018.1532818

Accepted author version posted online: 08

Oct 2018.

Submit your article to this journal

View Crossmark data 


\title{
Novel insights into the dynamics behavior of Glucagon-Like Peptide-1 Receptor with its small molecule Agonists
}

\author{
Khyati Girdharr $^{\# \$}$, Budheswar Dehury ${ }^{\sharp, \$,}$, Mahender Kumar Singh ${ }^{£}$, Vineeth P Daniel ${ }^{\#}$, Abhinav Choubey ${ }^{\#}$, Surbhi
} Dogra $^{\#}$, Sunil Kumar"ll, Prosenjit Mondal ${ }^{* \#}$

${ }^{\#}$ School of Basic Sciences, Indian Institute of Technology Mandi, Mandi-175001, H.P, India

${ }^{\ddagger}$ Biomedical Informatics Centre, ICMR-Regional Medical Research Centre, Chandrasekharpur, Bhubaneswar751023, Odisha, India

'Department of Chemistry, Technical University of Denmark, DTU Chemistry, DK-2800 Kongens Lyngby, Denmark (Present Address)

${ }^{\mathrm{E}}$ National Brain Research Centre, Manesar, Gurugram, Haryana-122051

IICAR-National Bureau of Agriculturally Important Microorganism, Kushmaur, Mau Nath Bhanjan -275103, U.P., India

${ }^{\$}$ These authors contributed equally to this work

Corresponding Authors

*E-mail: Prosenjit Mondal -prosenjit@iitmandi.ac.in, Phone No.+91-8894296497 


\begin{abstract}
The glucagon-like peptide-1 receptor (GLP-1R) is a well-known target of therapeutics industries for the treatment of various metabolic diseases like Type2 Diabetes and Obesity. The structural-functional relationships of small molecule agonists and GLP-1R are yet to be understood. Therefore, an attempt was made on structurally known GLP-1R agonists (Compound 1, Compound 2, Compound A, Compound B, and (S)-8) to study their interaction with the extracellular domain of GLP-1R. In this study, we explored the dynamics, intrinsic stability and binding mechanisms of these molecules through computational modeling, docking, molecular dynamics (MD) simulations and MM/PBSA binding free energy estimation. Molecular docking study depicted that hydrophobic interaction (pipi stacking) play a crucial role in maintaining the stability of the complex, which was also supported by intermolecular analysis from MD simulation study. Principal component analysis suggested that the terminal ends along with the turns/loops connecting adjacent helix and strands exhibits comparatively higher movement of main chain atoms in most of the complexes. MM/PBSA binding free energy study revealed that non-polar solvation (van der Waals and electrostatic) energy subsidizes significantly to the total binding energy and the polar solvation energy opposes the binding agonists to GLP-1R. Overall, we provide structural features information about GLP-1R complexes that would be conducive for the discovery of new GLP-1R agonists in the future for treatment of various metabolic diseases.
\end{abstract}

Key words: Agonists, docking, Type2 diabetes, GLP-1R, molecular dynamics 


\section{Introduction}

International Diabetes Federation (IDF) estimates that there are about approximately 64,200,000 individuals with Type 2 Diabetes Mellitus (T2DM) in India and by 2030 the number will reach to 100,000,000, as predicted by the World Health Organization (WHO) (Interantional Diabetes Federation, 2015).

T2DM is characterized by a deficit in "functional" beta-cell mass, resulting in insufficient insulin secretion to counter metabolic demands of insulin resistance and to maintain normoglycemia (Jensen, Cnop, Hull, Fujimoto, \& Kahn, 2002). To overcome the impaired beta-cells secretory response to glucose in T2DM patients as well as in subjects vulnerable to develop T2DM, it is desirable to introduce a therapeutic molecule in pancreatic beta-cells which can modulate insulin production and secretion. Numbers of therapeutics are available in the market for treatment of T2DM like metformin, sulfonylurea, thiazolidinedione, inhibitors or modulators of sodium-glucose cotransporter 2 (SGLT2), dipeptidyl peptidase 4 (DPP-4) and GLP-1R agonists (Yang, Zhou, Liu, \& Wang, 2015). Among all therapeutics, GLP-1R based therapy is a well-known promising approach in the treatment of diabetes by augmenting glucose-dependent insulin secretion with low risk of hypoglycemia. Incretin-based drugs like analogs of GLP-1 and its more stable analog exendin-4 (Ex-4) are being used to augment glucose-stimulated insulin secretion (GSIS) from beta-cells for better glycemic control in T2DM patients (Baggio \& Drucker, 2007; Drucker \& Nauck, 2006; Nielsen, Young, \& Parkes, 2004). GLP-1 exerts its effects after binding to its specific GLP-1R which belongs to B family of G-protein Coupled Receptor (GPCR), consists of the N-terminal extracellular domain and a transmembrane domain. The N-terminus extracellular domain of GPCR-B family is known to have a major role in the determination of the ligand binding (De Maturana, Willshaw, Kuntzsch, Rudolph, \& Donnelly, 2003). Cterminus of the GLP-1(endogenous agonist of GLP-1R) binds to the extracellular domain of receptor and help in stabilizing its binding while N-terminus binds to the transmembrane domain of the receptor (Santiago, Paci, \& Donnelly, 2018) and activates adenylyl cyclase thereby elevate cAMP (Furman, Ong, \& Pyne, 2010; Hansotia et al., 2004; Tengholm, 2012). However, GLP-1 has its own limitation with its administration and its short biological halflife in blood because of its metabolic stability and rapid degradation by DPP-4. The limitation of breakdown by DPP-IV is to some extent overcome by a GLP-1R agonist such as Ex-4 (Parkes, Mace, \& Trautmann, 2013) and Liraglutide (Gupta, 2013) (elinically approved GLP-1R peptide agonists for the treatment of diabetes) (Lotte B. Knudsen et al., 2000). However, the stability of these peptides, its synthesis, cost and its routes of administration is still a major challenge. Therefore, there is a need to identify and develop a small organic molecule that binds orthostatically and activate the receptor to resolve this issue. From the last few decades, there are very few small molecule GLP-1R agonists has been reported in which most potent agonist Boc5 and its analog WB4-24 show both antidiabetic and anti-obesity effect (Su et al., 2008).

Compound 1, Compound 2 (Lotte Bjerre Knudsen et al., 2007), Compound A, Compound B (Sloop et al., 2010) and (S)-8 (G. Morris \& Huey, 2009), all are GLP-1R allosteric modulators have been determined by high-throughput screening on the basis of cAMP and intracellular calcium mobilization. These compounds have known to act as allosteric activators of the receptor (Bueno et al., 2016; Koole et al., 2010; L. C. Morris et al., 2014). Methods that were used till now for the screening of above GLP-1R agonist are time-consuming and cost ineffective (Lotte Bjerre 
Knudsen et al., 2007; L. C. Morris et al., 2014; Sloop et al., 2010; M. W. Wang, Liu, \& Zhou, 2010). This might be one of the reasons that there is very few small molecules GLP-1R agonist reported so far. Therefore, for the development of potent GLP-1R allosteric agonists, it becomes a prime need to understand the interaction between the agonists and the receptor. Some of the reports try to mention the interaction of GLP-1R with its agonists (Lin \& Wang, 2009; Santiago et al., 2018) but till now there is no report on the study of an allosteric agonist of GLP-1R with the extracellular domain. Here in our report, we selected some of the small molecule GLP-1R agonists based on the Lipinski rule of five and tried to study their interaction with the extracellular domain of GLP-1R. In our study, AutoDock software was extensively used to determine the binding site of small molecules with GLP-1R followed by molecular dynamics (MD) simulation to inspect the dynamics and mode of binding of all agonists, Overall, the computational investigation of the extracellular domain of GLP-1R was carried out to open up a better understanding of the mechanism of binding GLP-1R agonists which would probably be an useful information for the development of potent GLP-1R agonist in near future.

\section{Materials and Methods}

\section{Preparation of Protein Structure}

The three-dimensional structure of the extracellular domain of GLP-1R in complex with GLP-1 peptide was retrieved from the RSCB Protein Data Bank [PDB Code: 3IOL]. Protein structure was prepared by removing GLP-1 peptide and water molecules associated with the crystal structure. Heteroatoms other than water molecules were also removed and the file was saved in PDB format and further used for the docking of reported agonists.

\section{Ligand selection and preparation}

The chemical structures of small molecules (Fig. 1) were retrieved as reported. From all reported GLP-1R agonist, 5 out of all are selected because of their reported allosteric property along with small molecules structure. Threedimensional PDB structures of all the agonists were obtained by using BIOVIA Discovery Studio (DS) Visualizer tool. The ligand molecules were then subjected to energy minimization through DS. Gasteiger charges were added to small molecules through AutoDock Tools.

\section{Molecular docking}

AutoDock 4.2.6 and AutoDock tools 1.5.6 (G. Morris \& Huey, 2009) were used to perform molecular docking, a computational docking program based on rapid Lamarckian genetic algorithm search method and empirical freeenergy force field (Forli et al., 2016). AutoDock Tools was utilized to prepare the input pdbqt file of GLP-1R [PDB: 3IOL]. Polar hydrogen atoms and Kollman charges were added to the 3D structure of GLP-1R. All torsional degrees of freedom, atomic partial charges, non-polar hydrogens and rotatable bonds were assigned to each ligand. The docking grid box of dimension $\mathrm{x}, \mathrm{y}$, and $\mathrm{z}$ are $102 \AA, 126 \AA$, and $104 \AA$ respectively with the spacing of $0.375 \AA$ was made to cover the entire extracellular GLP-1R domain. The best docking conformation was calculated by using the Lamarckian Genetic Algorithm (LGA) that is considered one of the best docking methods in AutoDock. The parameter used for LGA includes an initial population size of 150, number of energy evaluation of 250000, and 
maximum generation of 27000 were defined to find one best individual. Each of molecule was docked using 100 LGA runs. Final docked conformations of each molecule were clustered using $2 \AA$ root mean square deviation (RMSD).

\section{Evaluation and visualization of docked conformations}

The docked conformations of each ligand were ranked into clusters based on their binding energy and the best conformations were visually analyzed. Non-bonded interactions including hydrogen bond, Vander wall, pi-pi and other electrostatic interaction between docked ligand and GLP-1R was analyzed using DS.

\section{Molecular Dynamics Simulation}

To explore the stability and conformational flexibility of all the GLP-1R complex systems, MD simulations were performed as described previously (Dehury, Behera, \& Mahapatra, 2017). AnteChamber Python Parser interface (ACPYPE) was employed to parameterize the required topologies, atomic types, and charges of ligands. In this study, Amber99SB-ILDN and General Amber force fields were used for protein and ligand respectively in GROMACSv2018.2. All complexes were solvated with TIP3P water model. For each system, a cubic box-setting with a minimal distance of $10 \AA$ between the complex and edge of the box was further solvated using periodic boundary conditions. Each system was neutralized with counter ions of strength $0.15 \mathrm{M}\left(\mathrm{Na}^{+} / \mathrm{Cl}^{-}\right)$(summarized in Table S1). Then, electro-neutralized systems were subjected to energy minimization on a convergence threshold of 1 kcal $\mathrm{mol}^{-1} \AA$ achieved using a conjugate gradient algorithm. After energy minimization, all systems equilibrated using NVT and NPT ensemble for 1 nano-second (ns). Finally, a production runs of $100 \mathrm{~ns}$ with an integration time step of 0.2 ps were performed at a constant temperature (V-rescale method) and pressure (Parrinello-Rahman method) using the Leapfrog algorithm. To constrain all H-bonds during the equilibration LINCS algorithm was used, while, long-range ionic interactions approximated using the particle-mesh Ewald (PME) algorithm. Trajectory snapshots were stored at every 0.2 ps during the simulation period and $3 \mathrm{D}$ coordinate files harvested for postdynamic analysis.

\section{Trajectory Analysis}

To analyze the resultant trajectories, GROMACS analysis toolkit utilities were used to determine dynamics stability parameters including backbone RSMD, the radius of gyration (Rg), root mean square fluctuations (RMSF), and inter-molecular hydrogen bond (H-Bond) distribution for each complex system. The intermolecular H-bonds formed between specific amino acids residues and ligand atoms were determined gmx hbond utility tool with the donoracceptor set at a maximum of $0.35 \mathrm{~nm}$. VMD was employed to assess the evolution of the secondary structural elements in the proteins during MD simulations. The 2D graphs were plotted using Xmgrace (Grace 5.1.21; http://plasma-gate.weizmann.ac.il/Grace/) and DS was used for protein-ligand interaction analysis.

\subsection{Principal Component Analysis and Clustering}

Principal component analysis (PCA), a promising statistical approach, often separates the collective motions from the local dynamics to a small subset comprised of principal components (PCs) that defines the collective motion. To observe the collective motion of GLP-1R complexes, PCA was performed using built-in modules of GROMACS i.e., gmx covar and gmx anaeig. The covariance matrix was diagonalized to extract a set of eigenvectors and 
eigenvalues which reflected concert motion of the molecules. A set of eigenvectors of protein main chain atoms have been calculated, while coordinates of every 500 ps were used for porcupine plots. Cluster analysis of the resultant trajectories was performed using the method described by Daura and coworkers (Daura et al., 1999) using the gmx cluster built-in module of Gromacs. The GROMOS, RMSD conformational clustering algorithm with a cutoff of $0.2 \mathrm{~nm}$ was used to extract the maximally occupied clusters by taking into account the protein conformation with the lowermost RMSD to the centroid.

\section{MM/PBSA Binding Free Energy}

The binding energy of each GLP-1R-ligand complex (GLP-1R with its agonists) was determined using the g_mmpbsa tool which employs the molecular mechanics-based Poisson-Boltzmann Surface Area (MM/PBSA) approach. A total of 1000 snapshots structures extracted from $100 \mathrm{~ns}$ trajectory at equal interval of time for determining the binding energy $\left(\left(\Delta \mathrm{G}_{\text {bind }}\right)\right.$ using the followings sets of equation:

$\mathrm{G}_{\mathrm{bind}}=\mathrm{G}_{\text {complex }}-\left(\mathrm{G}_{\mathrm{GLP}-1 \mathrm{R}}+\mathrm{G}_{\text {ligand }}\right) \ldots \ldots \ldots \ldots$ (1)

$\mathrm{G}_{\text {bind }}=\mathrm{E}_{\text {gas }}+\mathrm{G}_{\mathrm{sol}}-\mathrm{T} \Delta \mathrm{S}$

$\mathrm{E}_{\text {gas }}=\mathrm{E}_{\text {int }}+\mathrm{E}_{\mathrm{vdw}}+\mathrm{E}_{\text {ele }}$

$\mathrm{G}_{\mathrm{sol}}=\mathrm{G}_{\mathrm{pol}}+\mathrm{GSA}$

$\mathrm{GSA}=\gamma \mathrm{SASA}+\mathrm{b}$

Absolute free energies of the GLP-1R-ligand complexes, receptor and ligands were denoted by $G_{\text {complex }}, G_{\text {GLP-1R }}$ and $\mathrm{G}_{\text {ligand }}$ respectively. The $\Delta \mathrm{G}_{\text {bind }}$ was decomposed to various terms; a gas-phase energy $\left(\mathrm{E}_{\mathrm{gas}}\right)$ can be defined as sum of bonded $\left(\mathrm{E}_{\mathrm{int}}\right)$ and non-bonded terms $\left(\mathrm{E}_{\mathrm{vdw}}\right.$ and $\left.\mathrm{E}_{\mathrm{ele}}\right)$; the solvation free energy $\left(\mathrm{G}_{\mathrm{sol}}\right)$ was decomposed into polar $\left(\mathrm{G}_{\mathrm{pol}}\right)$ and nonpolar (GSA) solvation energy components- and an entropy term (T $\Delta \mathrm{S})$. Polar solvation energies were determined by solving the Poisson-Boltzmann linear equation while nonpolar solvation through the solvent accessible surface area with an offset value (b) of $3.84928 \mathrm{kJmol}^{-1}$ and surface tension proportionality $(\gamma)$ set at $0.0226778 \mathrm{~kJ} \cdot \mathrm{mol}^{-1} \AA^{-2}$. A detailed description of methods used in the calculation can be found in the previous report (C. Wang, Greene, Xiao, Qi, \& Luo, 2018). The per-residue energy decomposition was also computed to understand the contribution of individual amino acids to the total binding energy.

\section{Results and Discussions}

\section{Molecular Docking}

Based on the structural motifs of the GLP-1R agonists, we divided them into three categories which include substituted quinoxaline, pyrimidine-based ago-allosteric modulators and pyridoindole based modulators (Graaf et al., 2016). Substituted quinoxaline derivative include Compound 1 and Compound 2; while, Compound A and Compound B/BETP belongs to the family of pyrimidine-based agonists and (S)-8 belong to tricyclic pyridoindolebased series of GLP-1R allosteric modulators (as shown in Fig. 1). All the selected agonist of GLP-1R have different structure motif, but, they all reported as positive agonist modulator of GLP-1R. The mode of binding of quinoxaline, pyrimidine and tricyclic pyridoindole agonists of GLP-1R is still unknown. Hence, to identify the binding sites involved in the allosteric interactions in between the extracellular domain of GLP-1R and abovementioned agonists, molecular docking was performed. 

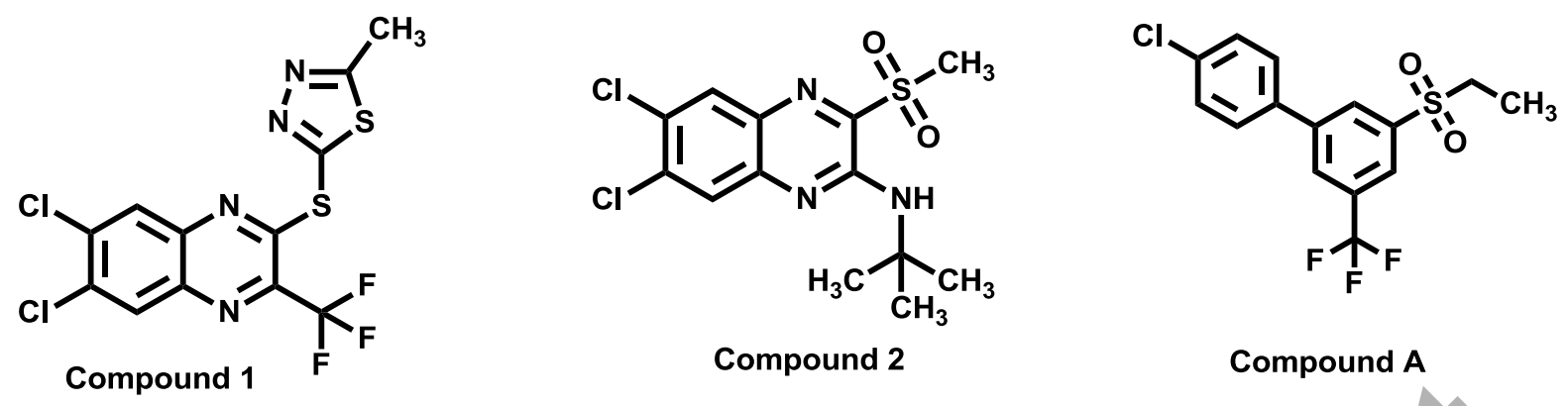

Compound $2 \quad$ Compound A
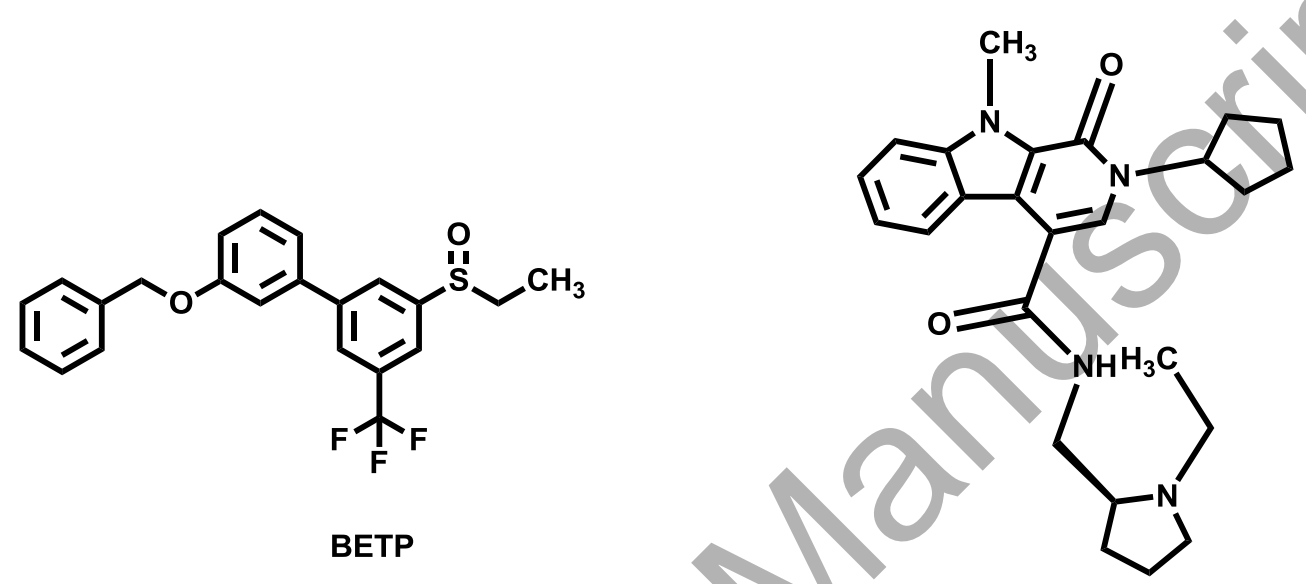

(S)-8

Fig 1: Chemical structure of small molecule GLP-1R agonists 
Molecular docking is mostly used for identification of binding affinities of a small molecule with the target protein (Lee, Jo, Lim, \& Im, 2012). In AutoDock tool, docking score is determined in terms of binding affinity in $\mathrm{kcal} / \mathrm{mol}$ (Lu et al., 2010). Binding energy is the sum of intermolecular energy (comprised of Hydrogen bond energy,

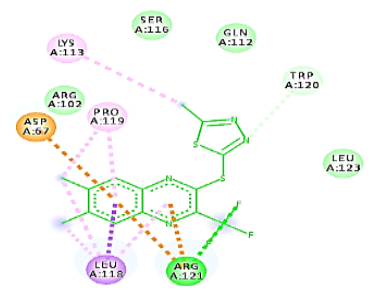

Compound 1

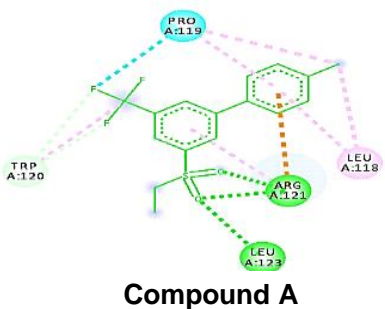

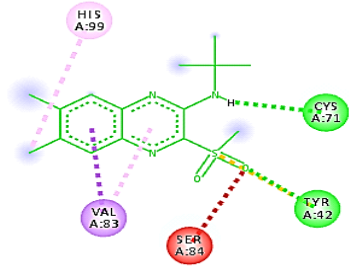

Compound 2

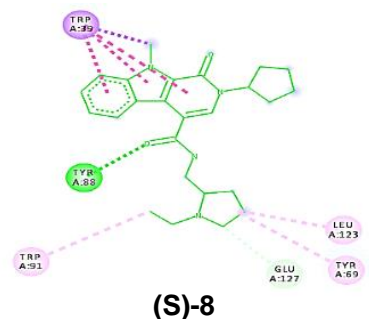

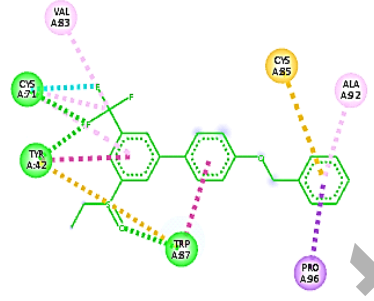

BETP

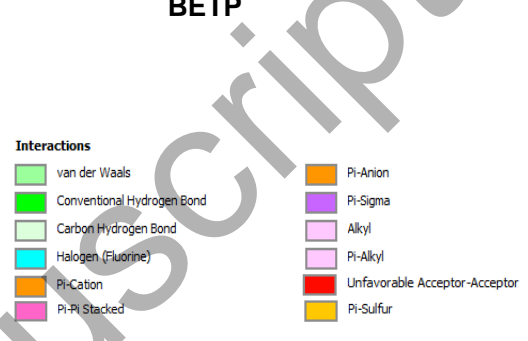

electrostatic energy, desolvation energy, and van der Wall energy) and torsional energy. A detailed summary of highest binding affinity, low inhibitory constant, no. of hydrogen bond formed and essential binding sites of all the above-mentioned compounds is presented in Table 1. The small molecule docking results were evaluated depending on their binding affinity, binding site and no. of H-Bond formed. The hundred docking conformations for each agonist were grouped according to their RMSD value of $2 \AA$. After docking, the obtained lower energy cluster conformations of each molecule were compared with the literature. The binding energy of (S)-8 and BETP was observed to be higher as compared to Compound 1, Compound 2 and Compound A as mentioned in Table 1. After performing docking study for each ligand molecules, the best pose (based on its conformation), binding sites and docking energy were determined. The binding site of the extracellular domain of GLP-1R and the involvement of estimated binding interactions of all the 5-agonists with GLP-1R, were mentioned in Fig. 2 and Table 1.

Fig 2: 2-Dimensional representation of binding sites of all docked GLP-1R agonists 
Table 1: Binding energy of GLP-1R with the extracellular domain of GLP-1R

\begin{tabular}{|c|c|c|c|c|c|}
\hline \multirow[t]{2}{*}{ Group } & \multirow[t]{2}{*}{ Compound } & \multirow{2}{*}{$\begin{array}{l}\text { Binding Energy } \\
\text { (kcal/mol) }\end{array}$} & \multirow{2}{*}{$\begin{array}{l}\text { Inhibitory } \\
\text { Constant }\end{array}$} & \multicolumn{2}{|c|}{ Hydrogen bond } \\
\hline & & & & Residue & Bond \\
\hline \multirow[t]{4}{*}{ Quinoxaline } & Compound 1 & $\begin{array}{l}-6.83 \\
\end{array}$ & $9.87 \mu \mathrm{M}$ & Arg121 & 2 \\
\hline & Compound 2 & -6.65 & $13.25 \mu \mathrm{M}$ & Tyr42 & 3 \\
\hline & & & & Cys71 & \\
\hline & & & & Ser84 & \\
\hline \multirow[t]{2}{*}{ Pyrimidine } & Compound A & -6.93 & $8.39 \mu \mathrm{M}$ & $\operatorname{Arg} 121$ & 2 \\
\hline & BETP & -7.95 & $1.49 \mu \mathrm{M}$ & Trp87 & \\
\hline Pyridoindole & (S)-8 & -8.38 & $717.98 \mathrm{nM}$ & Tyr88 & \\
\hline
\end{tabular}

The docking conformations and binding energies of Compound 1, Compound 2, Compound A, BETP and (S)-8 were compared with the experimental data of all reported agonists as determined previously (Wootten et al., 2013). Wootten and co-workers have described the differential activation of GLP-1R-by-GLP-1R agonists (BOC5, TT15, and BMS21) and allosteric modulator (Compound 2 and BETP). Their data suggests BETP has more potent allosteric activity than Compound 2 in enhancement of biased signaling. After docking of all the agonists, it is observed that the presence of trifluoromethyl group in Compound 1 increased its binding with receptor by forming 2-Hydrogen bonds with Arg121, while, Asp67 is found to stabilize the binding mode by forming $\pi$-sigma and $\pi$ anion interaction. Compound 1 showed maximum hydrophobic interactions with amino acid residues like Leu123, Gln112, Ser116 and Arg112, while the binding site and binding residues of another quinoxaline derivative, Compound 2 were different from Compound 1 . Val72 residue stabilize the Compound 2 by forming both $\pi$-sigma and $\pi$-alkyl interaction with the quinoxaline ring of Compound 2. Compound 2 is also observed to form three hydrogen bonds, in which two H-bonds are formed with Tyr42 and Ser84 due to the presence of the sulphonyl group in Compound 2, while the third H-bond was formed to be with Cys71. There was also an unfavorable anionicanionic interaction observed between sulphonyl group of Compound 2 and Ser84 of GLP-1R.

Compound A and BETP both being pyrimidine-based ago-allosteric modulators, show differential binding sites. BETP binding seemed to be more favorable than Compound 2 due to $\pi-\pi$ stacking interaction with Trp 87 and Tyr 42 amino acid residues. Nevertheless, due to the presence of trifluoromethyl and sulphonyl group, BETP showed three hydrogen bond interaction, one of which the shortest hydrogen bond was with Trp87 while the other two were weak hydrogen bond with Tyr42 and Cys72. Pro96 and Cys85 also interacted with BETP by $\pi$-sigma and $\pi$-Sulphur interactions. Compound A lacked $\pi-\pi$ stacking and showed only two hydrogen bond formation with Arg121 and Leu123. Weak interactions like $\pi$-cationic and halogen interaction made Compound A, a weak agonist of GLP-1R, which in turn favors the experimental data (Sloop et al., 2010).

(S)-8 compound is also observed to have both pi-pi stacking and hydrogen bond formation with higher binding energy. Trp39 residue of the extracellular GLP-1R show pi-pi stacking and pi-sigma interaction with (S)-8. The hydrogen bonding with residue Tyr88 made the interaction stronger. Other than these strong interactions, some of 
the weaker $\pi$-alkyl and alkyl interactions with Trp91, Tyr69 and Leu123 were also found to present in GLP-1R- (S)8 complex (Fig. 2).

The obtained docking results of all the five structurally diverse agonists of GLP-1R give us knowledge about the binding mode and affinity of the ligand which in results allow us to purpose novel binding residues involved in the activation of GLP-1R. Compound 2 and BETP has been experimentally reported to be a good agonist of GLP-1R which totally favors our docking results as Compound 2 shows weak allosteric binding site in our docking results which make us interpret the binding of Compound 2 to be probably at orthosteric binding site. Compound 2 has been reported more potent in the enhancement of intracellular cAMP than Compound 1, which may be due to the formation of a maximum number of the hydrogen bond or due to sharing of the orthosteric binding site by Compound 2. The experimental study on Compound 1 as an allosteric ligand has not reported so far, our data suggests high allosteric activity of Compound 1 than Compound 2. BETP also have the ability to act via multiple pathways through GLP-1R e.g., cAMP, pERK1/2, iCa, $\beta$-Arr-1 and $\beta$-Arr-2. To some extent, this may be explained by comparing the binding energy of BETP with other ligands and strongest $\pi$ - $\pi$ interaction formed by BETP. From all the above data, it may be concluded that amino acid residue Tyr42, Cys71 and Val83 is mainly involved in the activation of the GLP-1R receptor. Other than these residual levels receptor activation, $\pi-\pi$ interaction with some of the amino acid residue like Tyr42 and Trp39 can also stabilize the receptor ligand binding and help in activation of different pathways. The results obtained from our study co-relate with the study performed by Wootten and coworkers' states Compound 2 to have less allosteric activity in presence of Oxyntomodulin (GLP-1 agonist) than BETP which can be predicted by high cAMP and $\mathrm{Ca}^{2+}$ concentration. Compound 2 seems to be a weak binder on an extracellular domain which explains its binding with the orthosteric site than the extracellular regions. All the above mentioned results suggest the binding behavior of concerned agonists with GLP-1R and the difference in the binding mode or site may explain the distinct functional profiles of these agonists. This knowledge of the appropriate binding site on receptor provide us the opportunity to target physiological important responses which can extend in to drug development strategies.

\section{Analysis of MD trajectory of GLP-1R complexes}

MD simulations study was conducted for the five complex systems to understand the structural and dynamic behavior of the GLP-1R when coupled with agonists. The intrinsic dynamic stability of each complex was investigated by backbone RMSD, Rg, and C $\alpha$ RMSF as a function of simulation time. RMSD measures the difference between the backbones of a protein from its initial structural conformation to its final position. The stability of the protein/enzymes/receptor-ligand complex relative to its conformation can be assessed by plotting the deviations produced during the course of the simulation. The smaller the deviations, the more stable the protein structure. RMSD value for the backbone was calculated for $100 \mathrm{~ns}$ simulation in order to check for the stability of all the systems. Close inspection of all the systems showed a stable trend in RMSD (with an average deviation of $\sim 0.19$ $\mathrm{nm})$ profile demonstrating the stable nature of the systems considered in the present study. Although GLP1-RCompound A system was found show little higher RMSD up to $80 \mathrm{~ns}$, but, later it attained equilibrium with a stable RMSD of $\sim 0.23 \mathrm{~nm}$ (Fig. 3A). Overall, most of the systems showed least backbone deviation indicating the stable 
nature of all the systems. In addition, an attempt was also made to compute the RMSD of ligands from the $100 \mathrm{~ns}$ MD trajectories (as shown in Fig. S1). Analysis of the ligand RMSD suggested that most of ligands showed least RMSD throughout the simulation signifying that the ligand slightly orients within the binding pocket of GLP1-R, which is due to the flexibility of ligand binding residues in the allosteric binding sites. Differences in the efficacy between GLP-1R and its agonists have been observed in several head-to-head trials but till now there are no explanations for their behaviors (Trujillo, Nuffer, \& Ellis, 2015). Another possible reason may be due to the variances in the agonist's structure and size leads to different efficacy of the agonists towards GLP-1R. Like the RMSD, $\mathrm{Rg}$ (which assess the compactness of the system) also portrayed a compact gyradius of $\sim 1.51 \mathrm{~nm}$ suggesting a consistent shape and size of all the systems during simulation (Fig. 3B). Direct insight into the structural fluctuation and flexibility of proteins can be assessed using RMSF of $\mathrm{C} \alpha$ atoms (Fig. 3C). The residue-based RMSF of profile for the all the system portrays more residues that are flexible at residue positioned at both the ends i.e., Nterminal and C-terminal. In addition, the residue positioned adjacent to the $\alpha 1$ helix i.e., turns at 54-58 amino acids along with turns/loops connecting adjacent $\beta$-strands displayed high peak indicating the flexibility of these residues. These changes in C $\alpha$-RMSF might be due to the flexibilities of these residues towards various ligands indicates flexible nature of the protein. Overall, most of the systems followed same trend in RMSF with negligible differences. A graphical representation of secondary structure analysis for the all the five GLP1-R complex MD simulations was shown in Fig. S2 indicates the integrity of the helix and strands were retained during MD simulation.

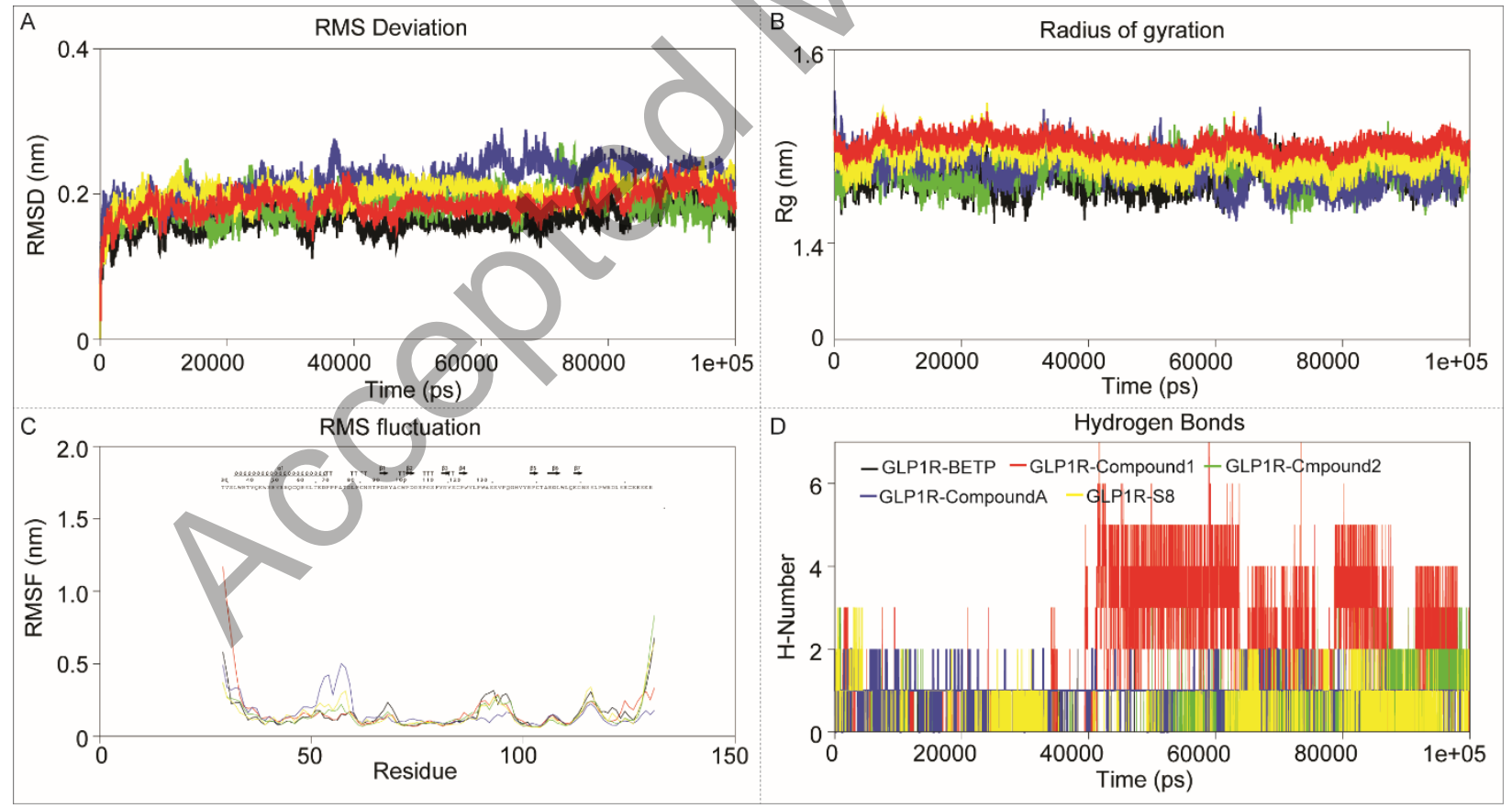

Fig. 3: Dynamics stability parameters of GLP-1R complexes over $100 \mathrm{~ns}$ MD simulation in aqueous solution. (A): Time dependences of root mean square deviation (RMSD) profile of backbone atoms of complexes during $100 \mathrm{~ns}$ MD simulations. (B): The conformational analysis of complexes displaying the radius of gyration over $100 \mathrm{~ns}$ MD simulations. (C): The C $\alpha$-RMSF profile of complexes during MD simulation. (D): Analysis of variation of 
intermolecular H-bonds of GLP-1R complexes during 100 ns MD simulation (Black: GLP-1R-BETP complex, Red: GLP-1R-Compound 1 complex, Green: GLP-1R-Compound 2 complex, Blue: GLP-1R-Compound A complex, and Yellow: GLP-1R-(S)-8 complex).

\section{Intermolecular H-bond and clustering analysis}

The stability of a protein and its binding components can be measured by the intermolecular H-bonds. In the GLP1R-BETP complex, an average of $\sim 0.98$ numbers H-bonds was observed for each frame as a function of simulation time; whereas, comparatively higher numbers of H-bonds ( 3.87) was noticed in the GLP-1R-Compound 1 complex (Fig. 3D). In contrast, GLP-1R-Compound A complex displayed an average of $\sim 2.08$ number of H-bonds for each frame throughout the simulation period followed by GLP-1R-(S)-8 complex with $\sim 1.87$ bonds. Complex with compound A displayed an average of $\sim 1.59$ numbers of H-bonds during $100 \mathrm{~ns}$ MD. Comparative analysis portrayed that in all complexes differential H-bonding pattern was observed, which might be due to the preferential affinity of GLP-1R towards each ligand within the active pocket and/or due to the slight change in ligand orientation within the ligand-binding pocket. To explore the conformational heterogeneity in the ensemble of GLP-1R-agonist structures generated from the MD simulations, clustering approach was employed using GROMOS clustering algorithm with a C $\alpha$-RMSD cut-off of $0.20 \mathrm{~nm}$. A total of 10,12, 14, 9 and 11 dominant clusters were obtained for BETP, Compound 1, Compound 2, Compound A and (S)-8 complexes from the $100 \mathrm{~ns}$ MD trajectories. The average RMSD of the clusters were found to be $0.24 \mathrm{~nm}, 0.20 \mathrm{~nm}, 0.23 \mathrm{~nm}, 0.28 \mathrm{~nm}$ and $0.21 \mathrm{~nm}$ respectively for BETP, Compound 1 , Compound 2, Compound A and (S)-8 complexes. Comparative analysis of the top ranked two clusters displayed that in all complexes, the dominant interaction was mediated by conserved hydrophobic contacts with few H-bonds (Fig. 4) which perfectly corroborate with the findings of molecular docking.

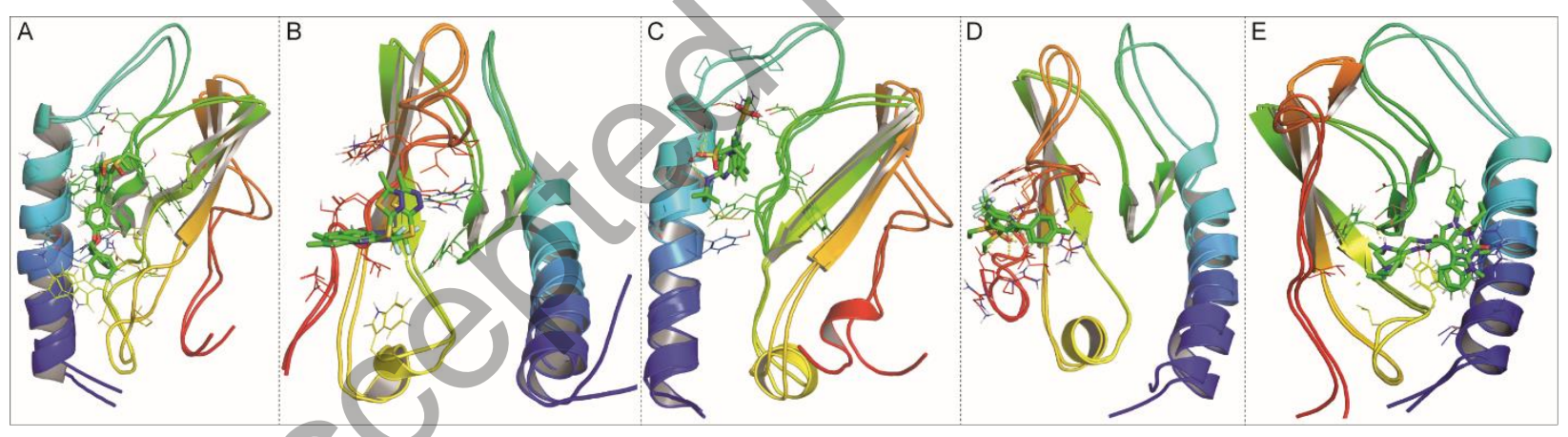

Fig. 4: The clustering analysis of the GLP1-R-ligand complexes. The top ranked two dominant clusters with ligands bound to GLP1-R obtained from trajectory have been displayed using DS.

\section{Essential dynamics (ED)}

The dynamic behavior of different components within biological macromolecules comprised of thermal fluctuations and collective motions, which may be related directly to their function. Therefore, PCA was performed using ED algorithms to filter the most prominent motions in the models generated from the less correlated ones (to elucidate intrinsic flexibility) relevant to protein function. The local or global changes are pivotal to apprehend the complex formational modulations in proteins (David \& Jacobs, 2014). Diagonal covariance matrices were built over the main chain atoms of each complex trajectory. The majority of protein dynamics can be successfully described by the first few eigenvectors (EVs) or principal components (PCs) of the entire system (Amadei, Linssen, \& Berendsen, 1993) 
the global movement of the backbone atoms of GLP-1R (in complex form) was calculated by analyzing the eigenvalues. The eigenvalues were obtained by the diagonalizing the covariance matrix of the $\mathrm{C} \alpha$ atomic fluctuations against the equivalent eigenvector indices (Fig. 5A). Precisely, the first two eigenvectors (EV1 and EV2) acquired $92.3 \%, 84.7 \%, 89.3 \%, 91.4 \%$ and $86.1 \%$ of the total motion in BETP, Compound 1, Compound 2, Compound A and (S)-8 complexes, demonstrating that these vectors describe the crucial subspace of the system. The scatter plot generated all complex systems (shown in Fig. 5B) indicated a substantial difference between GLP1R ligand complexes, which was well supported by porcupine plot (Fig. 6). As evident from 2D projection plot (Fig. 5B), it can be observed that both GLP-1R-Compound 1 and GLP-1R-(S)-8 complexes displayed the comparatively higher movement of main chain atoms (both in phase space and structurally) than the other complexes. The trace values of covariance matrixes of the complexes were found to be 7.61, 10.3, 6.59, 8.84 and $12.51 \mathrm{~nm}^{2}$ for GLP-1RBETP, GLP-1R-Compound 1, GLP-1R Compound 2, GLP-1R-Compound A and GLP-1R-(S)-8 complexes respectively. The overall motion between the all the five systems, as shown by the plot was found be more or less same in nature with minor changes, which might be due to the preferential binding of ligand within the binding cavity of agonists to GLP-1R. The porcupine plots were constructed to visualize the movements graphically using the structural coordinates of the EV1 for each complex, as shown in Fig. 6. It was observed that in all complexes, the majority of the motions were due to the contribution of residues of terminal ends along with the loops/turns adjoining the helix and beta strands.

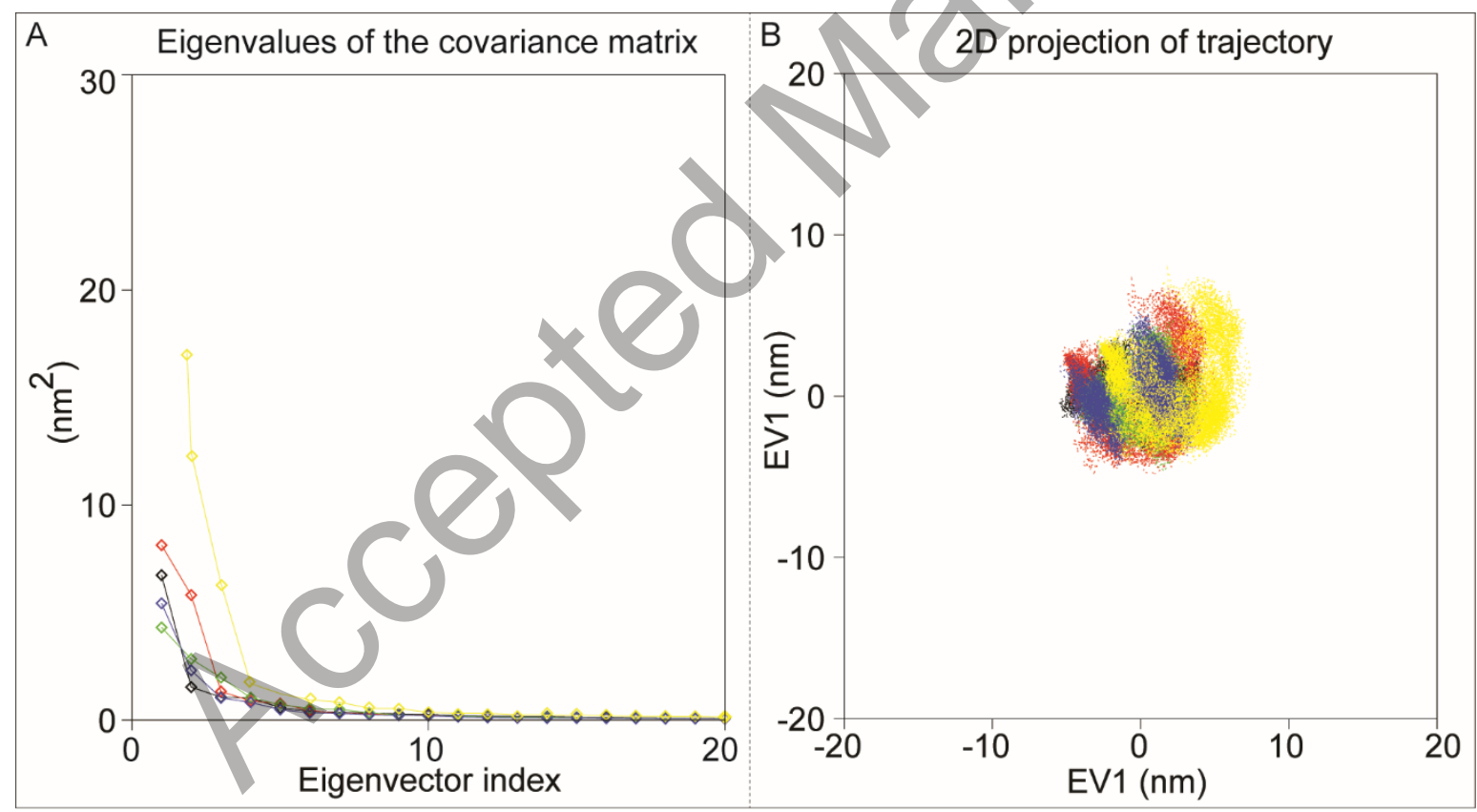

Fig. 5: Principal component analysis of GLP-1R complexes. (A): Comparison of eigenvalues plotted against the corresponding eigenvector indices coming from the $\mathrm{C} \alpha$ covariance matrix constructed form the equilibrium phase of MD simulations. (B) The 2D projection of each GLP-1R complex over in phase space along the first two principal eigenvectors components during 100 ns MD (Black: GLP-1R-BETP complex, Red: GLP-1R-Compound 1 complex, Green: GLP-1R-Compound 2 complex, Blue: GLP-1R-Compound A complex, and Yellow: GLP-1R-(S)-8 complex). 


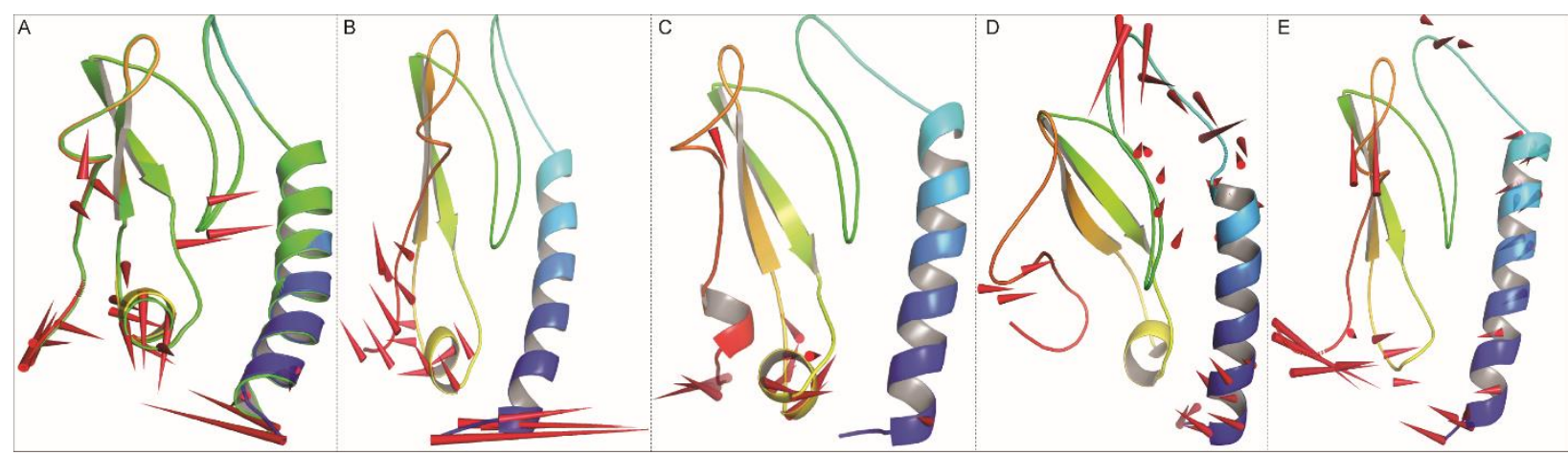

Fig. 6: Prevalent motions in the GLP-1R-agonist complexes using principal component analysis. The Porcupine plot of the first eigenvector generated through PCA of the representative structures of GLP-1R complexes. The vectors, represented as arrows, show the tendency of movement (A: GLP-1R-BETP complex, B: GLP-1R-Compound 1 complex, C: GLP-1R-Compound 2 complex, D: GLP-1R-Compound A complex, and E: GLP-1R-(S)-8 complex).

\section{Binding Free Energy Calculation}

MD trajectories of all the GLP-1R complexes were used to calculate binding free energy using MM/PBSA method. In recent years, this method has been applied various studies including conformer stability, protein-ligand interactions, protein design, and re-scoring (Genheden \& Ryde, 2015; C. Wang et al., 2018). Depending on the composition of ligands, different interactions ranging from hydrophobic, hydrogen, electrostatics and pi-pi interactions were formed within GLP-1R complexes. In this study, 1000 snapshots were extracted at equal intervals from the $100 \mathrm{~ns}$ MD trajectories to compute binding free energy for each complex. Each of these individual interactions contributes either positively or negatively to the overall binding free energy. The different energy terms contributing to the binding free energy of each complex has been summarized in Table 2. The estimated binding energies for each of these complexes were within the range of $-30.62 \pm 1.67$ to $-106.78 \pm 3.31 \mathrm{kJmol}^{-1}$. As evident from the Table 2, it can be observed that GLP-1R-Compound 1 complex possess least negative binding energy ($\left.30.62 \pm 1.67 \mathrm{kJmol}^{-1}\right)$, whereas, GLP-1R-BETP complex displayed portrayed highest free energy $(-106.78 \pm 3.31$ $\mathrm{kJmol}^{-1}$ ). Further discrete energy terms contributing towards binding free energy revealed that the polar solvation energy opposes the binding of agonists to GLP-1R and non-polar solvation energy subsidized significantly to the total binding energy (Table 2). Among the various energy terms, solvent-accessible surface area (SASA) energy $\left(\Delta \mathrm{G}_{\text {sasa }}\right)$ contributes least towards negative binding free energy (within the range of $-6.05 \pm 0.38$ to $-17.45 \pm 0.42$ $\mathrm{kJmol}^{-1}$ ). The van der Waals and electrostatic interaction were found to be relatively higher than the other energy term $\Delta \mathrm{G}_{\text {sasa }}$. From MM/PBSA calculations and the interaction plot generated by DS, it can be observed that hydrophobic interactions play a pivotal role in the stability of GLP-1R complexes. High negative value of vdW energy signifies the considerable hydrophobic interaction exists between GLP-1R and its agonists (well supported by molecular interaction analysis shown in Fig. 7 and Table S2-6). In this study, we employed quantum mechanics approach to compute the binding affinity of ligands towards GLP-1R receptor. The quantum mechanical (QM) methods offer the accurate depiction of molecular structures, dynamics, and functions. However, in case of complex biochemical systems and/or biochemical processes that are too long, the classical approaches serve as one of the pivotal tools due their efficiency and equitable accuracy. Among the different classical methods, the MM/PBSA 
approach has materialized as an efficient and reliable method to understand crucial molecular recognition processes (Kollman et al., 2000; C. Wang et al., 2018). Moreover, this method has been often a choice for efficient and reliable calculation of free energy of protein-ligand and protein-peptide and protein-protein interactions. For simple and small systems, the free energy perturbation and thermodynamic integration methods (developed much earlier than the MM/PBSA) shown to be more accurate than the MM/PBSA method. Due to low efficiency and slow convergence, their applications to typically large and complex molecular recognition processes are relatively limited. MM/PBSA methods have been used in wide spectrum of applications to estimate the binding free energies at a reasonable computational cost. The applications of the MMPBSA method have grown considerably in many different areas of bio-molecular study including protein-ligand binding affinity calculations due to their utility in drug discovery research. At present due to lack of experimental evidence on the binding energy, we cannot correlate our computed energy of the ligand towards receptor. However, our study has portrayed the energetic component that drives the receptor-ligand interaction at molecular scale.

Table 2: MM/PBSA binding free energies calculated using the g_mmpbsa method. Various energy terms contributing to the total free energy of GLP-1R ligand complexes

\begin{tabular}{|c|c|c|c|c|c|}
\hline Energetic components $(\mathrm{kJ} / \mathrm{mol})$ & BETP & Compound 1 & Compound 2 & Compound A & (S)-8 \\
\hline van der Waal energy $\left(\Delta G_{v w d}\right)$ & $-183.03 \pm 4.31$ & $-58.21 \pm 3.44$ & $-64.12 \pm 3.78$ & $-67.00 \pm 3.51$ & $-58.44 \pm 3.76$ \\
\hline Electrostatic energy $\left(\Delta \mathrm{G}_{\mathrm{ele}}\right)$ & $-67.31 \pm 1.71$ & $-70.39 \pm 4.59$ & $-58.95 \pm 3.57$ & $-174.99 \pm 9.32$ & $-134.79 \pm 6.03$ \\
\hline Polar solvation energy $\left(\Delta \mathrm{G}_{\mathrm{ps}}\right)$ & $160.68 \pm 3.34$ & $104.86 \pm 7.29$ & $96.27 \pm 6.62$ & $179.01 \pm 15.53$ & $97.10 \pm 7.81$ \\
\hline SASA energy $\left(\Delta \mathrm{G}_{\text {saas }}\right)$ & $-17.45 \pm 0.42$ & $-6.83 \pm 0.40$ & $-6.59 \pm 0.40$ & $-9.04 \pm 0.49$ & $-6.05 \pm 0.38$ \\
\hline Binding energy $\left(\Delta \mathrm{G}_{\text {binding }}\right)$ & $-106.78 \pm 3.31$ & $-30.62 \pm 1.67$ & $-33.67 \pm 1.69$ & $-72.19 \pm 2.31$ & $-100.63 \pm 3.48$ \\
\hline
\end{tabular}

\section{Intermolecular contact analysis before and after MD}

To understand the intermolecular contacts between agonists with the crystal structure of GLP-1R, a comparative analysis was performed before and post MD simulation (top ranked obtained from clustering) for each complex (as shown in Table S2-S6). In case of GLP-1R-BETP complex, the dominant binding attractions were by Leu50 and Trp87 residues where they contributed more by forming H-bonds rather than hydrophobic and halogen contacts with agonist BETP molecule. The Pi-Sulphur, Alkyl, and Pi-Alkyl contacts were found among the hydrophobic contacts. Further, decomposition the binding energy into different energy terms on a pre-residue basis revealed that the electrostatic energy, vdW, polar and non-polar solvation energy terms of the above residues contribute significantly to the binding energy. Post MD analysis of GLP-1R-Compound 1 complex portrayed three H-bonds formed by $\operatorname{Arg} 121$, and Leu123 with an average distance of $\sim 2.4 \AA$. Compound 2 shows more of hydrophobic interaction than the hydrogen bonds. Ser49 is the only residue which form hydrogen bond with Compound 2 Post-MD simulation. Like, GLP-1R-Compound 1 complex, H-bonds, hydrophobic and alkyl contacts favor the binding of Compound 2 with the receptor. In contrast to the previous complexes, the GLP-1R-Compound A complex was dominated by three H-bonds formed by Trp120, Leu123 and Arg131. In addition to strong H-bonds, the compound was well stabilized though one electrostatic and 4 hydrophobic (alkyl and pi-alkyl) contacts. In contrast to all, GLP-1R-(S)-8 complex displayed 3 H-bond formed by Glu68 and Trp39 residues, and 8 hydrophobic contacts (Pi-Pi Stacked and 
Pi-Alkyl) (Table S6). Furthermore, we have also computed the energetic contribution of each residue towards negative free energy (as shown Fig. S3). As evident from Fig. S3, it can be observed that most of the ligand binding residues significantly contribute to the overall negative free energy. Altogether, the results of intermolecular contact analysis perfectly corroborate with the contribution of different energetic terms i.e., vdW and non-polar solvation energies favorable for binding.

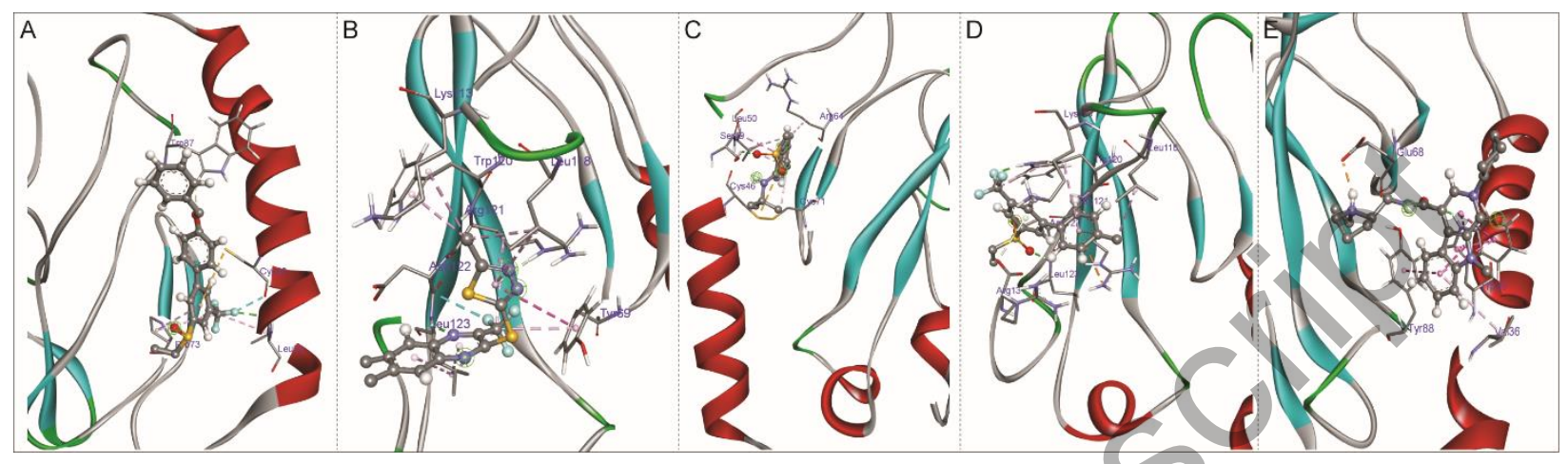

Fig. 7 Molecular interaction of the agonist's with GLP1-R receptor obtained from clustering analysis (Agonists displayed in ball and stick model, interacting residues in stick model along with the receptor colored by secondary structure elements)

\section{Conclusion}

In the present study, we have performed molecular docking of five structurally known diverse GLP-1R agonists. The docking studies revealed the significance of variable binding sites of these agonists and critical amino acid residues involved in the recognition of these agonists. MD simulations of the complexes of GLP-1R with all five different agonists were performed to understand the dynamics behavior of these complexes at the molecular scale. Our results suggested that all the agonists could exactly bind to the active pocket of GLP-1R to display agonistic activity. Similar to the reported data of BETP and Compound 2, our data suggests a probable high agonist activity of (S)-8 in comparison to experimentally validated Compound 2 (Lotte Bjerre Knudsen et al., 2007; L. C. Morris et al., 2014). However, there is no experimental evidence for (S)-8 in comparison with BETP and Compound 2. Our results also presented higher agonist activity of the above-mentioned compounds to be driven due to more hydrophobic interactions of agonists which the receptor protein. In our report, we have tried to describe all the possible allosteric interactions between the five-small molecule GLP-1R agonists with extracellular domain of GLP$1 \mathrm{R}$ along with its probable explanation for their differential experimental behavior. It is our expectations, mapping all these possible interacting avenues between GLP-1R and its agonists will significantly broaden our understanding of regulatory mechanisms within the GLP-1R and its agonist's axis. In turn, this will provide new strategies to screen and identify novel insulin secretagogue compounds having a greater efficacy of glucose-dependent insulinotropic action and restoration of islet glucosensitivity with improved intrinsic protection against hypoglycemia without compromising the efficacy of treatment. In summary, we have demonstrated the agonist binding mechanism of GLP-1R, which can be useful to develop other agonist-based receptor models in order to 
answer imperative questions associated with structure to function, and to propose agonist-mediated receptor activation mechanisms in near future.

\section{Conflicts of interest}

No potential conflicts of interest relevant to this article were reported.

\section{Acknowledgments}

We sincerely thank the BioX Centre of IIT Mandi for the facility and this work was partially supported by Seed Research grant from IIT Mandi and Khyati thanks the UGC, India for her fellowship.

\section{Author Contributions}

$\mathrm{KG}$ and $\mathrm{BD}$ conducted the majority of the experiments and performed data analyses. MKS performed part of the molecular dynamic simulation and SK reviewed the manuscript. PM designed study, and supervised the project; KG, BD, VD, AC, SD, SK and PM wrote the manuscript. PM is the guarantor of this work and, as such, had full access to all the data in the study and takes responsibility for the integrity of the data and the accuracy of the data analysis.

\section{References}

Amadei, A., Linssen, A. B. M., \& Berendsen, H. J. C. (1993). Essential dynamics of proteins. Proteins: Structure, Function, and Bioinformatics. https://doi.org/10.1002/prot.340170408

Baggio, L. L., \& Drucker, D. J. (2007). Biology of Incretins: GLP-1 and GIP. Gastroenterology. https://doi.org/10.1053/j.gastro.2007.03.054

Bueno, A. B., Showalter, A. D., Wainscott, D. B., Stutsman, C., Marín, A., Ficorilli, J., ... Sloop, K. W. (2016). Positive allosteric modulation of the glucagon-like peptide-1 receptor by diverse electrophiles. Journal of Biological Chemistry. https://doi.org/10.1074/jbc.M115.696039

Daura, X., Gademann, K., Jaun, B., Seebach, D., van Gunsteren, W. F., \& Mark, A. E. (1999). Peptide Folding: When Simulation Meets Experiment. Angewandte Chemie International Edition. https://doi.org/10.1002/(SICI)1521-3773(19990115)38:1/2<236::AID-ANIE236>3.3.CO;2-D

David, C. C., \& Jacobs, D. J. (2014). Principal component analysis: a method for determining the essential dynamics of proteins. Methods in Molecular Biology (Clifton, N.J.). https://doi.org/10.1007/978-1-62703-658-0_11

De Maturana, R. L., Willshaw, A., Kuntzsch, A., Rudolph, R., \& Donnelly, D. (2003). The isolated N-terminal domain of the glucagon-like peptide-1 (GLP-1) receptor binds exendin peptides with much higher affinity than GLP-1. Journal of Biological Chemistry, 278(12), 10195-10200. https://doi.org/10.1074/jbc.M212147200

Dehury, B., Behera, S. K., \& Mahapatra, N. (2017). Structural dynamics of Casein Kinase I (CKI) from malarial parasite Plasmodium falciparum (Isolate 3D7): Insights from theoretical modelling and molecular simulations. Journal of Molecular Graphics and Modelling. https://doi.org/10.1016/j.jmgm.2016.11.012

Drucker, D. J., \& Nauck, M. A. (2006). The incretin system: glucagon-like peptide-1 receptor agonists and 
dipeptidyl peptidase-4 inhibitors in type 2 diabetes. Lancet. https://doi.org/10.1016/S0140-6736(06)69705-5

Forli, S., Huey, R., Pique, M. E., Sanner, M. F., Goodsell, D. S., \& Olson, A. J. (2016). Computational proteinligand docking and virtual drug screening with the AutoDock suite. Nature Protocols. https://doi.org/10.1038/nprot.2016.051

Furman, B., Ong, W. K., \& Pyne, N. J. (2010). Cyclic AMP signaling in pancreatic islets. Advances in Experimental Medicine and Biology. https://doi.org/10.1007/978-90-481-3271-3_13

Genheden, S., \& Ryde, U. (2015). The MM/PBSA and MM/GBSA methods to estimate ligand-binding affinities. Expert Opinion on Drug Discovery. https://doi.org/10.1517/17460441.2015.1032936

Graaf, C. de, Donnelly, D., Wootten, D., Lau, J., Sexton, P. M., Miller, L. J., ... Wang, M.-W. (2016). GlucagonLike Peptide-1 and Its Class B G Protein-Coupled Receptors: A Long March to Therapeutic Successes. Pharmacological Reviews. https://doi.org/10.1124/pr.115.011395

Gupta, V. (2013). Glucagon-like peptide-1 analogues: An overview. Indian Journal of Endocrinology and Metabolism. https://doi.org/10.4103/2230-8210.111625

Hansotia, T., Baggio, L. L., Delmeire, D., Hinke, S. A., Yamada, Y., Tsukiyama, K., ... Drucker, D. J. (2004). Double Incretin Receptor Knockout (DIRKO) Mice Reveal an Essential Role for the Enteroinsular Axis in Transducing the Glucoregulatory Actions of DPP-IV Inhibitors. Diabetes. https://doi.org/10.2337/diabetes.53.5.1326

Interantional Diabetes Federation. (2015). IDF Diabetes Atlas. International Diabetes Federation. https://doi.org/10.1289/image.ehp.v119.i03

Jensen, C. C., Cnop, M., Hull, R. L., Fujimoto, W. Y., \& Kahn, S. E. (2002). beta-cell function is a major contributor to oral glucose tolerance in high-risk relatives of four ethnic groups in the U.S. Diabetes. https://doi.org/10.2337/diabetes.51.7.2170

Knudsen, L. B., Kiel, D., Teng, M., Behrens, C., Bhumralkar, D., Kodra, J. T., ... Lau, J. (2007). Small-molecule agonists for the glucagon-like peptide 1 receptor. Proceedings of the National Academy of Sciences of the United States of America. https://doi.org/10.1073/pnas.0605701104

Knudsen, L. B., Nielsen, P. F., Huusfeldt, P. O., Johansen, N. L., Madsen, K., Pedersen, F. Z., ... Agersø, H. (2000). Potent derivatives of glucagon-like peptide-1 with pharmacokinetic properties suitable for once daily administration. Journal of Medicinal Chemistry. https://doi.org/10.1021/jm9909645

Kollman, P. A., Massova, I., Reyes, C., Kuhn, B., Huo, S., Chong, L., ... Cheatham, T. E. (2000). Calculating structures and free energies of complex molecules: Combining molecular mechanics and continuum models. Accounts of Chemical Research. https://doi.org/10.1021/ar000033j

Koole, C., Wootten, D., Simms, J., Valant, C., Sridhar, R., Woodman, O. L., ... Sexton, P. M. (2010). Allosteric 
Ligands of the Glucagon-Like Peptide 1 Receptor (GLP-1R) Differentially Modulate Endogenous and Exogenous Peptide Responses in a Pathway-Selective Manner: Implications for Drug Screening. Molecular Pharmacology. https://doi.org/10.1124/mol.110.065664

Lee, H. S., Jo, S., Lim, H. S., \& Im, W. (2012). Application of binding free energy calculations to prediction of binding modes and affinities of MDM2 and MDMX inhibitors. Journal of Chemical Information and Modeling. https://doi.org/10.1021/ci3000997

Lin, F., \& Wang, R. (2009). Molecular modeling of the three-dimensional structure of GLP-1R and its interactions with several agonists. Journal of Molecular Modeling. https://doi.org/10.1007/s00894-008-0372-2

Lu, S. Y., Jiang, Y. J., Lv, J., Wu, T. X., Yu, Q. Sen, \& Zhu, W. L. (2010). Molecular docking and molecular dynamics simulation studies of GPR40 receptor-agonist interactions. Journal of Molecular Graphics and Modelling. https://doi.org/10.1016/j.jmgm.2010.02.001

Morris, G., \& Huey, R. (2009). AutoDock4 and AutoDockTools4: Automated docking with selective receptor flexibility. Journal of .... https://doi.org/10.1002/jcc.21256.AutoDock4

Morris, L. C., Nance, K. D., Gentry, P. R., Days, E. L., Weaver, C. D., Niswender, C. M., ... Lindsley, C. W. (2014). Discovery of (S)-2-cyclopentyl-N-((1-isopropylpyrrolidin2-yl)-9-methyl-1-oxo-2,9-dihydro-1Hpyrrido[3,4-b]indole-4-carboxamide (VU0453379): a novel, CNS penetrant glucagon-like peptide 1 receptor (GLP-1R) positive allosteric modulator (PAM). Journal of Medicinal Chemistry. https://doi.org/10.1021/jm501375c

Nielsen, L. L., Young, A. A., \& Parkes, D. G. (2004). Pharmacology of exenatide (synthetic exendin-4): A potential therapeutic for improved glycemic control of type 2 diabetes. Regulatory Peptides. https://doi.org/10.1016/j.regpep.2003.10.028

Parkes, D. G., Mace, K. F., \& Trautmann, M. E. (2013). Discovery and development of exenatide: the first antidiabetic agent to leverage the multiple benefits of the incretin hormone, GLP-1. Expert Opinion on Drug Discovery. https://doi.org/10.1517/17460441.2013.741580

Santiago, C. G., Paci, E., \& Donnelly, D. (2018). A mechanism for agonist activation of the glucagon-like Peptide-1 (GLP-1) receptor through modelling \& molecular dynamics. Biochemical and Biophysical Research Communications, 498(2), 359-365. https://doi.org/10.1016/j.bbrc.2018.01.110

Sloop, K. W., Willard, F. S., Brenner, M. B., Ficorilli, J., Valasek, K., Showalter, A. D., .. Coghlan, M. J. (2010). Novel small molecule glucagon-like peptide-1 receptor agonist stimulates insulin secretion in rodents and from human islets. Diabetes. https://doi.org/10.2337/db10-0689

Su, H., He, M., Li, H., Liu, Q., Wang, J., Wang, Y., .. Wang, M. W. (2008). Boc5, a non-peptidic glucagon-like peptide-1 receptor agonist, invokes sustained glycemic control and weight loss in diabetic mice. PLoS ONE. https://doi.org/10.1371/journal.pone.0002892 
Tengholm, A. (2012). Cyclic AMP dynamics in the pancreatic $\beta$-cell. Upsala Journal of Medical Sciences. https://doi.org/10.3109/03009734.2012.724732

Trujillo, J. M., Nuffer, W., \& Ellis, S. L. (2015). GLP-1 receptor agonists: A review of head-to-head clinical studies. Therapeutic Advances in Endocrinology and Metabolism. https://doi.org/10.1177/2042018814559725

Wang, C., Greene, D., Xiao, L., Qi, R., \& Luo, R. (2018). Recent Developments and Applications of the MMPBSA Method. Frontiers in Molecular Biosciences. https://doi.org/10.3389/fmolb.2017.00087

Wang, M. W., Liu, Q., \& Zhou, C. H. (2010). Non-peptidic glucose-like peptide-1 receptor agonists: Aftermath of a serendipitous discovery. Acta Pharmacologica Sinica. https://doi.org/10.1038/aps.2010.107

Wootten, D., Savage, E. E., Willard, F. S., Bueno, A. B., Sloop, K. W., Christopoulos, A., \& Sexton, P. M. (2013). Differential Activation and Modulation of the Glucagon-Like Peptide-1 Receptor by Small Molecule Ligands. Molecular Pharmacology. https://doi.org/10.1124/mol.112.084525

Yang, D. H., Zhou, C. H., Liu, Q., \& Wang, M. W. (2015). Landmark studies on the glucagon subfamily of GPCRs: From small molecule modulators to a crystal structure. Acta Pharmacologica Sinica. https://doi.org/10.1038/aps.2015.78

Amadei, A., Linssen, A. B. M., \& Berendsen, H. J. C. (1993). Essential dynamics of proteins. Proteins: Structure, Function, and Bioinformatics. https://doi.org/10.1002/prot.340170408

Baggio, L. L., \& Drucker, D. J. (2007). Biology of Incretins: GLP-1 and GIP. Gastroenterology. https://doi.org/10.1053/j.gastro.2007.03.054

Bueno, A. B., Showalter, A. D., Wainscott, D. B., Stutsman, C., Marín, A., Ficorilli, J., ... Sloop, K. W. (2016). Positive allosteric modulation of the glucagon-like peptide-1 receptor by diverse electrophiles. Journal of Biological Chemistry. https://doi.org/10.1074/jbc.M115.696039

Daura, X., Gademann, K., Jaun, B., Seebach, D., van Gunsteren, W. F., \& Mark, A. E. (1999). Peptide Folding: When Simulation Meets Experiment. Angewandte Chemie International Edition. https://doi.org/10.1002/(SICI)1521-3773(19990115)38:1/2<236::AID-ANIE236>3.3.CO;2-D

David, C. C., \& Jacobs, D. J. (2014). Principal component analysis: a method for determining the essential dynamics of proteins. Methods in Molecular Biology (Clifton, N.J.). https://doi.org/10.1007/978-1-62703-658-0_11

De Maturana, R. L., Willshaw, A., Kuntzsch, A., Rudolph, R., \& Donnelly, D. (2003). The isolated N-terminal domain of the glucagon-like peptide-1 (GLP-1) receptor binds exendin peptides with much higher affinity than GLP-1. Journal of Biological Chemistry, 278(12), 10195-10200. https://doi.org/10.1074/jbc.M212147200

Dehury, B., Behera, S. K., \& Mahapatra, N. (2017). Structural dynamics of Casein Kinase I (CKI) from malarial 
parasite Plasmodium falciparum (Isolate 3D7): Insights from theoretical modelling and molecular simulations. Journal of Molecular Graphics and Modelling. https://doi.org/10.1016/j.jmgm.2016.11.012

Drucker, D. J., \& Nauck, M. A. (2006). The incretin system: glucagon-like peptide-1 receptor agonists and dipeptidyl peptidase-4 inhibitors in type 2 diabetes. Lancet. https://doi.org/10.1016/S0140-6736(06)69705-5

Forli, S., Huey, R., Pique, M. E., Sanner, M. F., Goodsell, D. S., \& Olson, A. J. (2016). Computational proteinligand docking and virtual drug screening with the AutoDock suite. Nature Protocols. https://doi.org/10.1038/nprot.2016.051

Furman, B., Ong, W. K., \& Pyne, N. J. (2010). Cyclic AMP signaling in pancreatic islets. Advances in Experimental Medicine and Biology. https://doi.org/10.1007/978-90-481-3271-3_13

Genheden, S., \& Ryde, U. (2015). The MM/PBSA and MM/GBSA methods to estimate ligand-binding affinities. Expert Opinion on Drug Discovery. https://doi.org/10.1517/17460441.2015.1032936

Graaf, C. de, Donnelly, D., Wootten, D., Lau, J., Sexton, P. M., Miller, L. J., ... Wang, M.-W. (2016). GlucagonLike Peptide-1 and Its Class B G Protein-Coupled Receptors: A Long March to Therapeutic Successes. Pharmacological Reviews. https://doi.org/10.1124/pr.115.011395

Gupta, V. (2013). Glucagon-like peptide-1 analogues: An overview. Indian Journal of Endocrinology and Metabolism. https://doi.org/10.4103/2230-8210.111625

Hansotia, T., Baggio, L. L., Delmeire, D., Hinke, S. A., Yamada, Y., Tsukiyama, K., ... Drucker, D. J. (2004). Double Incretin Receptor Knockout (DIRKO) Mice Reveal an Essential Role for the Enteroinsular Axis in Transducing the Glucoregulatory Actions of DPP-IV Inhibitors. Diabetes. https://doi.org/10.2337/diabetes.53.5.1326

Interantional Diabetes Federation. (2015). IDF Diabetes Atlas. International Diabetes Federation. https://doi.org/10.1289/image.ehp.v119.i03

Jensen, C. C., Cnop, M., Hull, R. L., Fujimoto, W. Y., \& Kahn, S. E. (2002). beta-cell function is a major contributor to oral glucose tolerance in high-risk relatives of four ethnic groups in the U.S. Diabetes. https://doi.org/10.2337/diabetes.51.7.2170

Knudsen, L. B., Kiel, D., Teng, M., Behrens, C., Bhumralkar, D., Kodra, J. T., ... Lau, J. (2007). Small-molecule agonists for the glucagon-like peptide 1 receptor. Proceedings of the National Academy of Sciences of the United States of America. https://doi.org/10.1073/pnas.0605701104

Knudsen, L. B., Nielsen, P. F., Huusfeldt, P. O., Johansen, N. L., Madsen, K., Pedersen, F. Z., ... Agersø, H. (2000). Potent derivatives of glucagon-like peptide-1 with pharmacokinetic properties suitable for once daily administration. Journal of Medicinal Chemistry. https://doi.org/10.1021/jm9909645

Kollman, P. A., Massova, I., Reyes, C., Kuhn, B., Huo, S., Chong, L., ... Cheatham, T. E. (2000). Calculating 
structures and free energies of complex molecules: Combining molecular mechanics and continuum models. Accounts of Chemical Research. https://doi.org/10.1021/ar000033j

Koole, C., Wootten, D., Simms, J., Valant, C., Sridhar, R., Woodman, O. L., ... Sexton, P. M. (2010). Allosteric Ligands of the Glucagon-Like Peptide 1 Receptor (GLP-1R) Differentially Modulate Endogenous and Exogenous Peptide Responses in a Pathway-Selective Manner: Implications for Drug Screening. Molecular Pharmacology. https://doi.org/10.1124/mol.110.065664

Lee, H. S., Jo, S., Lim, H. S., \& Im, W. (2012). Application of binding free energy calculations to prediction of binding modes and affinities of MDM2 and MDMX inhibitors. Journal of Chemical Information and Modeling. https://doi.org/10.1021/ci3000997

Lin, F., \& Wang, R. (2009). Molecular modeling of the three-dimensional structure of GLP-1R and its interactions with several agonists. Journal of Molecular Modeling. https://doi.org/10.1007/s00894-008-0372-2

Lu, S. Y., Jiang, Y. J., Lv, J., Wu, T. X., Yu, Q. Sen, \& Zhu, W. L. (2010). Molecular docking and molecular dynamics simulation studies of GPR40 receptor-agonist interactions. Journal of Molecular Graphics and Modelling. https://doi.org/10.1016/j.jmgm.2010.02.001

Morris, G., \& Huey, R. (2009). AutoDock4 and AutoDockTools4: Automated docking with selective receptor flexibility. Journal of .... https://doi.org/10.1002/jcc.21256.AutoDock4

Morris, L. C., Nance, K. D., Gentry, P. R., Days, E. L., Weaver, C. D., Niswender, C. M., ... Lindsley, C. W. (2014). Discovery of (S)-2-cyclopentyl-N-((1-isopropylpyrrolidin2-yl)-9-methyl-1-oxo-2,9-dihydro-1Hpyrrido[3,4-b]indole-4-carboxamide (VU0453379): a novel, CNS penetrant glucagon-like peptide 1 receptor (GLP-1R) positive allosteric modulator (PAM). Journal of Medicinal Chemistry. https://doi.org/10.1021/jm501375e

Nielsen, L. L., Young, A. A., \& Parkes, D. G. (2004). Pharmacology of exenatide (synthetic exendin-4): A potential therapeutic for improved glycemic control of type 2 diabetes. Regulatory Peptides. https://doi.org/10.1016/j.regpep.2003.10.028

Parkes, D. G., Mace, K. F., \& Trautmann, M. E. (2013). Discovery and development of exenatide: the first antidiabetic agent to leverage the multiple benefits of the incretin hormone, GLP-1. Expert Opinion on Drug Discovery. https://doi.org/10.1517/17460441.2013.741580

Santiago, C. G., Paci, E., \& Donnelly, D. (2018). A mechanism for agonist activation of the glucagon-like Peptide-1 (GLP-1) receptor through modelling \& molecular dynamics. Biochemical and Biophysical Research Communications, 498(2), 359-365. https://doi.org/10.1016/j.bbrc.2018.01.110

Sloop, K. W., Willard, F. S., Brenner, M. B., Ficorilli, J., Valasek, K., Showalter, A. D., ... Coghlan, M. J. (2010). Novel small molecule glucagon-like peptide-1 receptor agonist stimulates insulin secretion in rodents and from human islets. Diabetes. https://doi.org/10.2337/db10-0689 
Su, H., He, M., Li, H., Liu, Q., Wang, J., Wang, Y., ... Wang, M. W. (2008). Boc5, a non-peptidic glucagon-like peptide-1 receptor agonist, invokes sustained glycemic control and weight loss in diabetic mice. PLoS ONE. https://doi.org/10.1371/journal.pone.0002892

Tengholm, A. (2012). Cyclic AMP dynamics in the pancreatic $\beta$-cell. Upsala Journal of Medical Sciences. https://doi.org/10.3109/03009734.2012.724732

Trujillo, J. M., Nuffer, W., \& Ellis, S. L. (2015). GLP-1 receptor agonists: A review of head-to-head clinical studies. Therapeutic Advances in Endocrinology and Metabolism. https://doi.org/10.1177/2042018814559725

Wang, C., Greene, D., Xiao, L., Qi, R., \& Luo, R. (2018). Recent Developments and Applications of the MMPBSA Method. Frontiers in Molecular Biosciences. https://doi.org/10.3389/fmolb.2017.00087

Wang, M. W., Liu, Q., \& Zhou, C. H. (2010). Non-peptidic glucose-like peptide-1 receptor agonists: Aftermath of a serendipitous discovery. Acta Pharmacologica Sinica. https://doi.org/10.1038/aps.2010.107

Wootten, D., Savage, E. E., Willard, F. S., Bueno, A. B., Sloop, K. W., Christopoulos, A., \& Sexton, P. M. (2013). Differential Activation and Modulation of the Glucagon-Like Peptide-1 Receptor by Small Molecule Ligands. Molecular Pharmacology. https://doi.org/10.1124/mol.112.084525

Yang, D. H., Zhou, C. H., Liu, Q., \& Wang, M. W. (2015). Landmark studies on the glucagon subfamily of GPCRs: From small molecule modulators to a crystal structure. Acta Pharmacologica Sinica. https://doi.org/10.1038/aps.2015.78 